\title{
IMPACT OF TIMING OF RENAL REPLACEMENT THERAPY INITIATION ON OUTCOME OF ACUTE KIDNEY INJURY IN SEPTIC PATIENTS
}

\author{
Muhammad Nasir, Madiha Hashmi, Muhammad Sohaib, Zahoor Ahmed, Muhammad Salman*, Syed Amir Raza \\ Aga Khan University, Karachi Pakistan, *Lyari General Hospital, Karachi Pakistan
}

\begin{abstract}
Objective: To identify whether the timing of initiation of continuous renal replacement therapy affects outcome in septic patients with acute kidney injury in term of 28 days mortality.

Study Design: Cross sectional analytical study.

Place and Duration of Study: This research was conducted at department of Anaesthesiology and critical care unit of the Aga Khan University Hospital, Karachi, from Oct 2018 to Jun 2019.

Methodology: The study reviewed all adult patients aged $>18$ years who developed acute kidney injury after septic shock and required continuous renal replacement therapy in surgical intensive care unit. Considering the value of blood urea nitrogen, patients were classified into two groups. One was in early group that's was defined as blood urea nitrogen value of $<100 \mathrm{mg} / \mathrm{dl}$ just before continuous renal replacement therapy initiation while the patients who have blood urea nitrogen value of $\geq 100 \mathrm{mg} / \mathrm{dl}$ just prior to continuous renal replacement therapy initiation were classified as late group. Kaplan-Meier survival analysis was performed and median survival was computed.

Results: Forty patients were included for analysis. There were thirty patients (75\%) in early group in whom continuous renal replacement therapy was started with mean blood urea nitrogen of $66 \pm 20.2 \mathrm{mg} / \mathrm{dL}$ and 10 $(25 \%)$ patients were in late group with mean blood urea nitrogen of $137 \pm 28.4 \mathrm{mg} / \mathrm{dL}$. The overall survival rates in both groups were $49.6 \%$, and $10.4 \%$ at 10 and 25 days, respectively. Median survival time was not statistically significant between early and late continuous renal replacement therapy groups 9 (2.74) vs. 11 (0.41); $p=0.997$.

Conclusion: Septic patients who developed acute kidney injury and started continuous renal replacement therapy did not showed in any mortality benefit among both groups.
\end{abstract}

Keywords: Acute kidney injury, Intensive care, Renal replacement therapy, Sepsis.

This is an Open Access article distributed under the terms of the Creative Commons Attribution License (http://creativecommons.org/licenses/by/4.0), which permits unrestricted use, distribution, and reproduction in any medium, provided the original work is properly cited.

\section{INTRODUCTION}

Acute kidney injury (AKI) is a common and devastating complication of critical illness. It is a common complication of intensive care unit ranging from $6 \%$ to $60 \%$ despite the technical advances and care in this field of medicine. Mortality remains very high in these group of patients $(50 \% \text { to } 63 \%)^{1}$. One of the studies conducted in Pakistan showed that $31 \%$ of patients admitted in critical care suffered AKI during the course of the disease ${ }^{2}$. Acute kidney injury is being one of the independent risk component for mortality and as well as the development of chronic renal failure in patients who suffered AKI in critical care ${ }^{3}$. The pathophysiology of AKI development after sepsis and septic shock is still unclear, but the leading

Correspondence: Dr Muhammad Nasir, Dept of Anesthesiology, Aga Khan University Karachi Pakistan

Received: 29 Oct 2019; revised received: 06 Dec 2020; accepted: 10 Jan 2021 cause of AKI in critically ill patients is sepsis and septic shock. Treatment of acute kidney injury is only supportive, most of the patient treats conservatively and interventions have not been benefit for restoration of renal function so it will not improve overall survival. Conventional hemodialysis requires hemodynamic stability. A lot of patients in intensive care units (ICU) are in septic shock and have unstable hemodynamics due to which they cannot undergo conventional dialysis. These patients can only benefit from continuous renal replacement therapy (CRRT). Severe AKI is mostly treated with specific modalities of hemodialysis that is continuous renal replacement therapy. If renal replacement therapy imitation started late then in-hospital mortality of such patients may exceeds up to $50 \%$. Early initiation of CRRT may be beneficial and improve survival that is explained by a recent meta-analysis, but most of 
the data were derived from observational studies $^{4}$. The review of the literature did not clearly explain the optimal time of continuous renal replacement therapy initiation in order to get maximum benefit in term of survival ${ }^{5}$. It is almost ten years that we have started CRRT at out center. This study helps us to find out the true efficacy of CRRT on our settings. Serum blood urea nitrogen (BUN) can be surrogate marker to early versus late initiation of continuous renal replacement therapy. The purpose of this study was to identify retrospectively whether the timing of initiation of continuous renal replacement therapy (CRRT) affects outcome in septic patients with acute renal failure (ARF) in term of 28 days mortality.

\section{METHODOLOGY}

Our hospital was commissioned in 1985. In 2009, the hospital had started CRRT in intensive care unit. Nephrologist and Intensivists were assigned for initiation of CRRT. The mechanism of data reporting is through electronic records by HIMS. We followed the departmental and hospital policy for access and referral of data. Ethical approval was obtained from the Ethics Review Committee of our University (Ref No: 2018-0430595 Dated October 22nd, 2018). We reviewed all patients who were admitted in Surgical ICU between 2009 to 2018 (10 years) with diagnosis of sepsis, severe sepsis and septic shock and developed acute kidney injury requiring CRRT. We designed a specific performa for the purpose of this audit. Patients who have CRRT duration less than 24 hours and having incomplete data were excluded. Patients, anes-thesiologist, primary physician and nurse' identification were not disclosed on the data collection form. Patient demographic details, comorbid, laboratory and clinical outcome data of patients who developed AKI and received CRRT as renal replacement therapy were noted. Considering the value of BUN we have classified patients into two groups. One wasearly group that's was defined as BUN value of $<100 \mathrm{mg} / \mathrm{dl}$ just before the start of CRRT while the patients with BUN value of $\geq 100 \mathrm{mg} / \mathrm{dl} \mathrm{just}$ prior to CRRT initiation were classified as Late group $^{6}$. AKI was defined on the basis of AKIN
(Acute Kidney Injury Network) criteria7. The definitions of the terms sepsis, severe sepsis and septic shock were taken from the society of critical care medicine criteria for the sepsis, severe sepsis and septic shock ${ }^{8}$. Admission glasgow coma scale (GCS) were noted while acute physiology and chronic health evaluation (APACHE II) were calculated after 24hours of admission. Other clinical parameters like urine output, 24 hours fluid balance just before start of RRT and vital signs on day of RRT initiation were noted. Primary outcome was the 28-day mortality following the start of RRT while secondary outcome were hospital length of stay (days) and duration of CRRT. Both outcomes were determined.

Data was analyzed by Statistical packages for social science version 20. Point estimation of statistics were reported in term of frequency, percentage, mean, standard deviation median survival according to quantitative and qualitative variables. Continuous and normally distributed variables were analyzed by student's t-test to compare mean difference between the groups while Mann-Whitney tests for non-normal data. Chi square test or fisher exact test was used to compare proportion difference between. Kaplan-Meier survival analysis was performed and median survival was computed. In all instances, the level of statistical significance was set at 0.05 .

\section{RESULTS}

A total of 46 patients were enrolled, who were admitted to the surgical intensive care unit with acute renal failure after septic shock requiring renal replacement therapy. Forty patients were selected for the analysis; Six patient were excluded due to missing data. The mean age of the patients was $55.30 \pm 15.91$ years (range: $28-81$ ). There were $26(65 \%)$ male and $14(35 \%)$ female. Thirty patients (75\%) with mean BUN value of 66 $\pm 20.2 \mathrm{mg} / \mathrm{dL}$ received early CRRT while only 10 patients $(25 \%)$ with BUN greater than 100 received late CRRT. The demographic characteristics of two groups are shown in table-I. The clinical parameters and laboratory data were comparable before the start of CRRT between the two groups 
as shown in table-II. Twenty-four hours urine output just prior to CRRT initiation was lower in the patients who had "early" CRRT initiation but statistically not significant. Between the two groups, no difference was found in APACHI II and Glasgow coma scale as shown in table-II. Mean Blood pressure, heart rate (HR) and respi-
In our study we were focusing on 28 days mortality between the early and late CRRT group but none of our patient survived beyond 25 days. The overall survival rates in both groups were $49.6 \%$, and $10.4 \%$ at 10 and 25 days, respectively as shown in the figure. Median survival time was not statistically significant between early and

Table-I: Demographic characteristics of patients.

\begin{tabular}{l|c|c|c}
\hline Variables & $\begin{array}{c}\text { Early } \\
\text { (BUN<100 } \mathbf{~ m / d L ) , ~} \mathbf{n = 3 0}\end{array}$ & $\begin{array}{c}\text { Late } \\
(\mathbf{B U N}>\mathbf{0 0} \mathbf{~ m g} / \mathbf{d L}), \mathbf{n}=\mathbf{1 0}\end{array}$ & $\boldsymbol{p}$-value \\
\hline Age (Years) & $51.47 \pm 15.53$ & $66.80 \pm 11.14$ & 0.007 \\
\hline Gender & \multicolumn{3}{|l}{} \\
\hline Male & $20(66.7 \%)$ & $6(60 \%)$ & 0.702 \\
Female & $10(33.3 \%)$ & $4(40 \%)$ & 0.067 \\
Pre-existent conditions & $14(46.7 \%)$ & $8(80 \%)$ & 0.673 \\
Diabetes Mellitus & $7(23.3 \%)$ & $3(30 \%)$ & 0.426 \\
Hypertension & $8(26.7 \%)$ & $4(40 \%)$ & 0.206 \\
IHD & $6(20 \%)$ & $4(40 \%)$ & 0.017 \\
Others & $6(20 \%)$ & $6(60 \%)$ &
\end{tabular}

Results are presented as mean \pm SD and frequency (\%).

Table-II: Clinical and laboratory parameter before crrt.

\begin{tabular}{|c|c|c|c|}
\hline Variables & $\begin{array}{c}\text { Early } \\
(\text { BUN }<100 \mathrm{mg} / \mathrm{dL}), \mathrm{n}=30\end{array}$ & $\begin{array}{c}\text { Late } \\
\text { (BUN>100 } \mathrm{mg} / \mathrm{dL}), \mathrm{n}=10\end{array}$ & $p$-value \\
\hline APACHI.II & $21.20 \pm 8.57$ & $26.80 \pm 3.05$ & 0.052 \\
\hline GCSon Admission & $13.13 \pm 2.58$ & $11.20 \pm 2.74$ & 0.050 \\
\hline Creatinine Admission (mg/dl) & $2.64 \pm 1.75$ & $5.64 \pm 5.35$ & 0.010 \\
\hline Fluids Admission 24hours & $2760 \pm 1011.52$ & $2130 \pm 616.53$ & 0.072 \\
\hline Hospital days of CRRT & $5.43 \pm 6.85$ & $6.60 \pm 5.72$ & 0.631 \\
\hline Urine Output & $303 \pm 277.29$ & $205 \pm 134.26$ & 0.292 \\
\hline Net Fluid balance & $2740 \pm 1046.37$ & $2270 \pm 749.89$ & 0.199 \\
\hline BUN prior CRT $(\mathrm{mg} / \mathrm{dl})$ & $53.20 \pm 23.33$ & $124.10 \pm 19.35$ & 0.001 \\
\hline Serum Creatinine prior $(\mathrm{mg} / \mathrm{dl})$ & $3.42 \pm 2.24$ & $6.57 \pm 2.34$ & 0.001 \\
\hline Heart rate (Beats/min) & $112.17 \pm 16.54$ & $109.50 \pm 15.71$ & 0.658 \\
\hline Mean blood pressure (mmHg) & $64.67 \pm 12.38$ & $62.60 \pm 12.83$ & 0.653 \\
\hline Respiratory Rate (Breaths/min) & $25.80 \pm 6.71$ & $29.40 \pm 6.11$ & 0.142 \\
\hline Vasopressor $\S$ & $26(86.7 \%)$ & $10(100 \%)$ & 0.224 \\
\hline
\end{tabular}

Results are presented as mean $\pm S D$ and $n(\%)$

ratory rate all were not statistically associated between the two groups. Hospital length of stay was little bit more in early group (13.83 \pm 13.66$)$ as compare to late $(12.70 \pm 8.73)$ but also not statistically significant $(p$-value $=0.81)$. Hospital days of CRRT duration in days were almost identical between the two groups with $p$-value of 0.63 as shown in table-II. CRRT duration in days was also high in early group $(3.50 \pm 2.1)$ as compare to late $(2.70 \pm 1.34)$ with $p$-value of 0.267 as shown in table-III. late CRRT groups 9 (2.74) vs. 11 (0.41); $p=0.997$. Survival rate in the "early" CRRT group was 70\% and $16.7 \%$ at 10 , and 25 days, respectively. The survival rates for the patients in the "late" CRRT group were $57.1 \%, 11.5 \%$ at 10 days and 25 days respectively as shown in figure.

\section{DISCUSSION}

Septic shock along with renal failure is associated with high mortality in critically ill patients. 
Several clinical efforts were done to determine ways to pick early deterioration in renal function so that clinical outcomes can be improved $^{9}$. There are a lot of anabolic and catabolic phenomena associated with end stage renal disease and these complicated metabolic derangements are treated by CRRT. Initiation of CRRT is associated with a lot of other factors that's includingthe patient's demographic data, socioeconomic status, and clinical conditions ${ }^{10,11}$. The question about to initiate dialysis in patient with AKI is still in debates, almost as long as

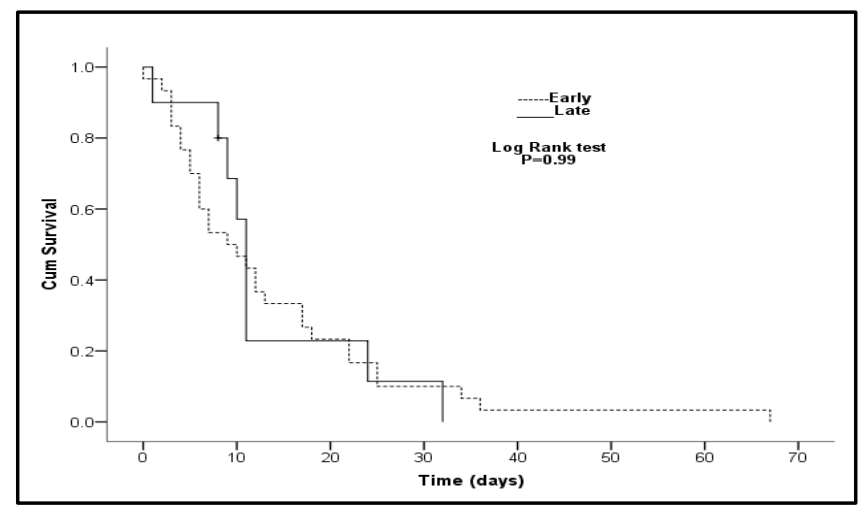

Figure: Kaplan-meir estimate of survival.

hemodialysis has been an integral part for treating patients with AKI in clinical medicines. Determining precise guidelines for commencing CRRT in patients with AKI is imperative; starting too early can lead to unnecessary risk associated with dialysis while on the other hand delayed treatment can have adverse outcomes. Hence clinical practices vary among the clinicians, so it has been too hard to decide when to initiate CRRT. RRT was initiated prophetically in 1950s. In 1960s and 1970s the literature showed that the quick beginning of RRT can improve survival ${ }^{12}$. Similar research data had been given in 2000s in cardiac surgery ${ }^{13}$, and oliguric septic patients ${ }^{11}$. Park et al, published prospective cohort study in 2016 showing the survival benefit in critically ill patients with AKI receiving early renal replacement therapy during the course of the disease ${ }^{11}$. In these different retrospective studies analyses the timing of the start of CRRT. BUN having delegated attention for the marker for the CRRT initiation by most of the studies, but only BUN is inadequate for assessing the renal function. It doesn't only affected by glomerular filtration rate but also by other mechanism like renal tubular function, protein intake and catabolism, numerus other important clinical situations like gastrointestinal bleeding and drugs ${ }^{14}$.

In this study, we determined the outcomes of CRRT in adult patients based on BUN value just prior to CRRT imitation. The results did not indicate a survival benefit of early CRRT initiation. Same findings are observed by prospective and retrospective studies done previously among critical ill patients in intensive care units requiring renal replacement therapy. Systematic review published in 2016 by Raymond et al, showed that no survival advantage was found with "early" RRT among high-quality studies with an OR of 0.665 (95\% CI 0.384-1.153, $p=0.146)^{17}$. Jee et al, conducted study (REANL study) in 2014 showing that earlier commencement of continuous renal replacement therapy was not associated with a significantly lower risk of death at 28 days ${ }^{15}$. Similar findings were observed in most of the studies showing no survival benefit with early CRRT15-20.

On the contrary there are studies showing survival benefit with early renal replacement therapy. Most of the retrospective studies argued that there is an increased survival rate association with early initiation of CRRT6,21,22. The study done by Carl et $a l^{6}$, included retrospective data on medical intensive care unit patients with sepsis and acute renal failure requiring renal replacement therapy. Renal replacement therapy started with a blood urea nitrogen (BUN) of $<100 \mathrm{mg}$ / dL was defined as "early" initiation, and initiation with a BUN $\geq 100 \mathrm{mg} / \mathrm{dL}$ was defined as "late" Upon logistic regression analysis, initiating dialysis with a BUN >100 mg/dL predicted death at 14 days (odds ratio [OR] 3.6, 95\% confidence interval $[\mathrm{CI}]$ 1.7-7.6, $p=0.001$ ), 28 days (OR 2.6, 95\% CI 1.2-5.7, $p=0.01$ ), and 365 days (OR 3.5, 95\% CI 1.2-10, $p=0.02)$. The study showed that septic patients who started on dialysis with BUN less than $100 \mathrm{mg} / \mathrm{dl}$ had improved mortality rates. 
In this study our all patients were in septic shock no one was in sepsis or in severe sepsis so our patients were more critically ill, in some previous retrospective studies severity of recruited patient were not too high 16,18 , this is the one of major strength of our study. According to the KDIGO Clinical Practice Guideline for acute kidneyinjury, the timingof RRT initiation was not clearly described it is dependent upon clinician's clinical decisions. So every author haphazardly defined early and late CRRT. In spite of the fact that RRT those started early may have better survival rate, but it is hard to suggest the proper timing for CRRT initiation. There are several limitations in this observational study. First, we didn't measure detail parameters of CRRT like choice and method of CRRT because in our study we have included very high-riskpatients those who were already in septic shock, so it was not easy to measure the detailed parameters of CRRT. On the contrary, it is also important to determine the modality of CRRT as it would be better considering the risks and benefits, clinically. Second, the sample size of this study was small and from one institute, so we could not generalize our results to various patient populations. There is need of some multicenter prospective study for future validation. Lastly, we did not analyze the specific indication for initiation of CRRT in septic patients with AKI. The reason of initiation of CRRT may have an impact on the prognosis of septic shock patients with AKI. The start of CRRT cannot be based on few variables; rather the whole clinical picture of patient is important in initiating the CRRT. In this study we tried to identify whether early or late initiation of CRRT in septic patients with AKI have any impact on mortality, but our study showed no difference between the two groups. There are limitations of this study which include small sample size and retrospective nature of study. Large prospective trails are needed to validate this study results.

\section{ACKNOWLEDGMENT}

I dedicate this article to all my teachers, faculties, nurses and health care providers those worked hard in intensive care unit and involved in the care of critically ill patients. I would also like to thank my family for always helping and comforting me.

\section{Funding Source}

This research received no grant from any funding agency in the public, commercial, or notfor-profit sectors.

\section{CONCLUSION}

No significant difference was found in patients' outcome who were put on CRRT for sepsis induced acute kidney injury. Timing of continuous renal replacement therapy (CRRT) does not affect the mortality in patient with AKI on basis of BUN value.

\section{CONFLICT OF INTEREST}

This study has no conflict of interest to be declared by any author.

\section{REFERENCES}

1. Wierstra BT, Kadri S, Alomar S, Burbano X, Barrisford GW, Kao RL. The impact of "early" versus "late" initiation of renal replacement therapy in critical care patients with acute kidney injury: a systematic review and evidence synthesis. Crit Care 2016; 20(1): 122-25.

2. Mansoor F, Akhtar A, Sikandar I, Qadeer A, Ali Z, Kaleem B. What factors on admission influence ICU mortality in adult patients admitted to the intensive care unit with severe pneumonia. Pak Armed Forces Med J 2016; 66(4): 548-52.

3. Hoste EA, Bagshaw SM, Bellomo R, Cely CM, Colman R, Cruz $\mathrm{DN}$, et al. Epidemiology of acute kidney injury in critically ill patients: the multinational AKI-EPI study. Crit Care Med 2015; 41(8): 1411-23.

4. Karvellas CJ, Farhat MR, Sajjad I, Mogensen SS, Leung AA, Wald R, et al. A comparison of early versus late initiation of renal replacement therapy in critically ill patients with acute kidney injury: a systematic review and meta-analysis. Crit Care 2011; 15(1): R72-75.

5. Zarbock A, Kellum JA, Schmidt C, Van Aken H, Wempe C, Pavenstädt $\mathrm{H}$, et al. Effect of early vs delayed initiation of renal replacement therapy on mortality in critically ill patients with acute kidney injury: the ELAIN randomized clinical trial. Jama 2016; 315(20): 2190-99.

6. Carl DE, Grossman C, Behnke M, Sessler CN, Gehr TW. Effect of timing of dialysis on mortality in critically ill, septic patients with acute renal failure. Hemodial Inter 2010; 14(1): 11-17.

7. Thomas ME, Blaine C, Dawnay A, Devonald MA, Ftouh S, Laing $C$, et al. The definition of acute kidney injury and its use in practice. Kidney Int 2015; 87(1): 62-73.

8. Singer M, Deutschman CS, Seymour CW, Shankar-Hari M, Annane D. The third international consensus definitions for sepsis and septic shock (Sepsis-3). Jama 2016; 315(8): 801-10.

9. Yoon BR, Leem AY, Park MS, Kim YS, Chung KS. Optimal timing of initiating continuous renal replacement therapy in septic shock patients with acute kidney injury. Sci Rep 2019; 9(1): 11981-85. 
10. Vasudevan A, Iyengar A, Phadke K. Modality of choice for renal replacement therapy for children with acute kidney injury: Results of a survey. Indian J Nephrol 2012; 22(2): 121-25.

11. Park JY, An JN, Jhee JH, Kim DK, Oh HJ, Kim S, et al. Early initiation of continuous renal replacement therapy improves survival of elderly patients with acute kidney injury: a multicenter prospective cohort study. Crit Care 2016; 20(1): 260-62.

12. Salisbury PF. Timely versus delayed use of the artificial kidney. AMA Arch Intern Med 1958; 101(4): 690-01.

13. Piccinni P, Dan M, Barbacini S, Carraro R, Lieta E, Marafon S, et al. Early isovolaemic haemofiltration in oliguric patients with septic shock. Intensive Care Med 2006; 32(1): 80-86.

14. Waikar SS, Bonventre JV. Can we rely on blood urea nitrogen as a biomarker to determine when to initiate dialysis. Am Soc Nephrol 2006: 1(5): 903-04.

15. Jun M, Bellomo R, Cass A, Gallagher M, Lo S, Lee J, et al. Timing of renal replacement therapy and patient outcomes in the randomized evaluation of normal versus augmented level of replacement therapy study. Crit Care Med 2014; 42(8): 1756-65.

16. Gaudry S, Hajage D, Schortgen F, Martin-Lefevre L, Pons B, Boulet $\mathrm{E}$, et al. Initiation strategies for renal-replacement therapy in the intensive care unit. N Engl J Med 2016; 375(2): 122-33.

17. Wierstra BT, Kadri S, Alomar S, Burbano X, Barrisford GW, Kao
RL. The impact of "early" versus "late" initiation of renal replacement therapy in critical care patients with acute kidney injury: a systematic review and evidence synthesis. Crit Care 2016; 20(1): 122-25.

18. Barbar SD, Clere-Jehl R, Bourredjem A, Hernu R, Montini F, Bruyere $\mathrm{R}$, et al. Timing of renal-replacement therapy in patients with acute kidney injury and sepsis. N Engl J Med 2018; 379(15): 1431-42.

19. Liu KD, Himmelfarb J, Paganini E, Ikizler TA, Soroko SH, Mehta RL. Timing of initiation of dialysis in critically ill patients with acute kidney injury. Clin J Am Soc Nephrol 2006; 1(5): 915-19.

20. Bagshaw SM, Uchino S, Bellomo R, Morimatsu H, Morgera S, Schetz $\mathrm{M}$, et al. Timing of renal replacement therapy and clinical outcomes in critically ill patients with severe acute kidney injury. J Crit Care 2009; 24(1): 129-40.

21. Chou YH, Huang TM, Wu VC, Wang CY, Shiao CC, Lai CF, et al. Impact of timing of renal replacement therapy initiation on outcome of septic acute kidney injury. Crit Care 2011; 15(3): R134.

22. Chon GR, Chang JW, Huh JW, Lim CM, Koh Y, Park SK, et al. A comparison of the time from sepsis to inception of continuous renal replacement therapy versus RIFLE criteria in patients with septic acute kidney injury. Shock 2012; 38(1): 30-36. 